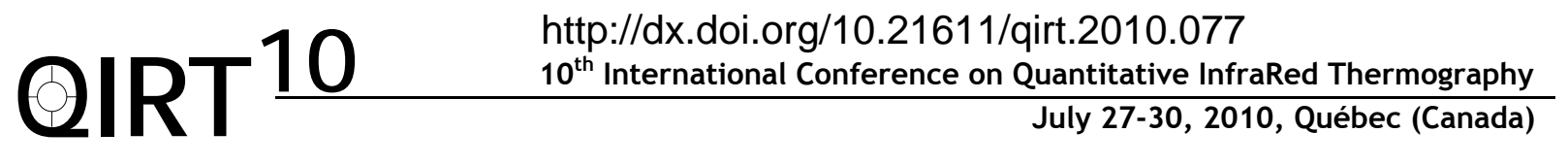

\title{
Thermal Sensitivity Analysis Applied on Infrared Thermography Evaluation
}

by F. R. Lopez*, and V. P. Nicolau*

*Mechanical Engineering Department, Federal University of Santa Catarina (Florianópolis), Santa Catarina, Brazil Campus Universitário - Trindade. E-mails: flopez@cwpanama.net, vicente@emc.ufsc.br

\section{Abstract}

There are many factors that affect the temperature distribution map of the surface being tested by Infrared Thermography. Internal defect properties such as thermal conductivity, heat capacity and defect depth, play an important role in the temperature behaviour of the pixels or regions being analyzed. Also is well known that other external factors such as the convection heat transfer, variation on the surface emissivity and ambient radiation reflectivity affects the thermographic signal received by the IR camera, as it's showed in figure 1.

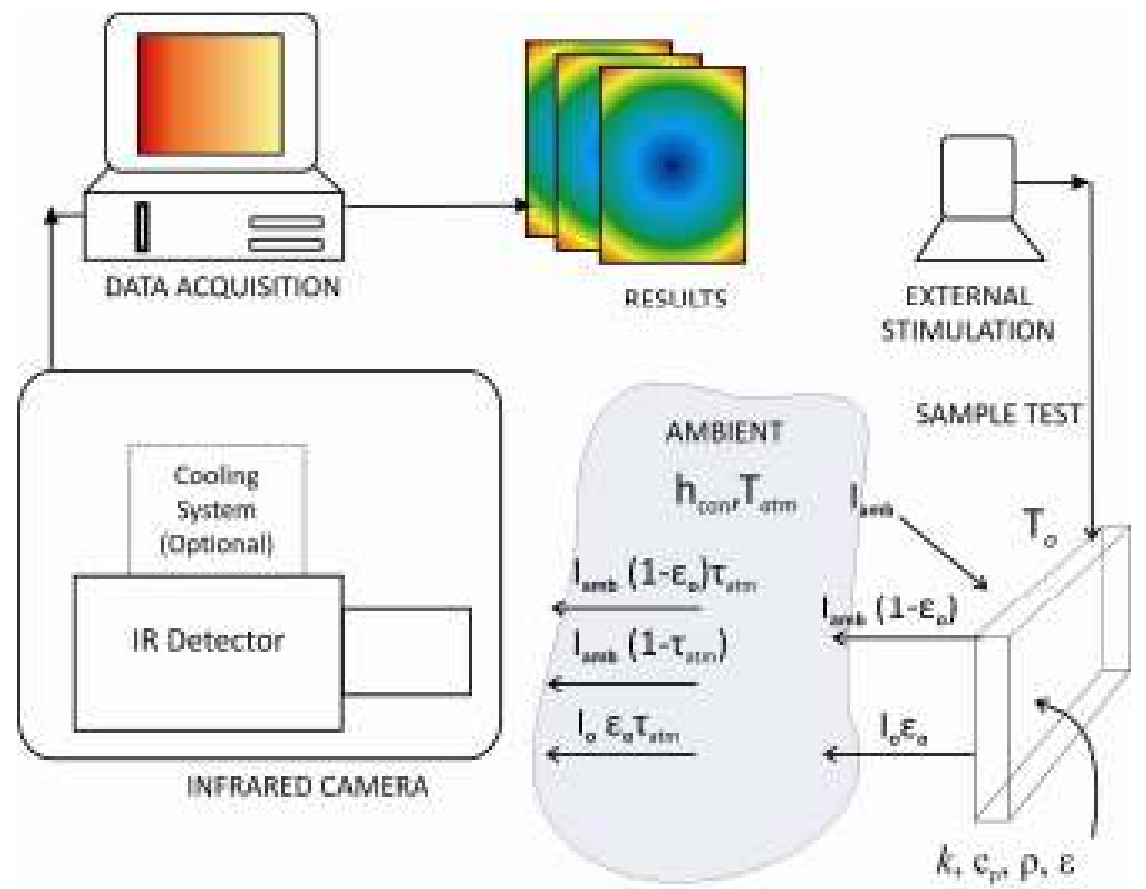

Fig. 1. Main actors during the thermographic inspection

The main objective of this work is to determine quantitatively how those parameters affect the thermal response of the surface being analyzed. Using a numerical model based on the transient 3D heat conduction equation, which describes the physics of the heat transfer process during the non-destructive test by infrared thermography, a sensitivity analysis is developed. The model consists in imposing a cooling process to the sample test, which starts from a high and uniform temperature, also considering the thermal transfer at the boundaries by convection and radiation [1]. The numerical solution of the model has been developed using the Finite Volume Method, which its approximated equations were obtained through the performing of energy balances on each elementary volumes.

The sensitivity analysis developed on this work consists in determining the Sensitivity Matrix or Jacobian, which in explicit form is written as:

$$
J(P)=\left[\frac{\partial T^{T}(P)}{\partial P}\right]
$$


The elements of the Jacobian are called the sensitivity coefficients $\mathrm{J}_{\mathrm{ij}}$, and they are a measure of the sensitivity of the estimated temperature $T_{i}$ with respect to perturbations in the parameter $P_{j}$ [2]. For determining the sensitivity coefficients was used finite difference approximation. As it's showed in figures $2 a, 2 b$ and $2 c$, temperature is more sensitive to changes in the parameter depth, followed by the thermal conductivity and heat capacity, respectively. These sensitivity coefficients were calculated for defect of air at different depth. Contributions pursuits in this work are the evaluation of defect thermal properties and external ambient conditions on the IR image during the non-destructive test.

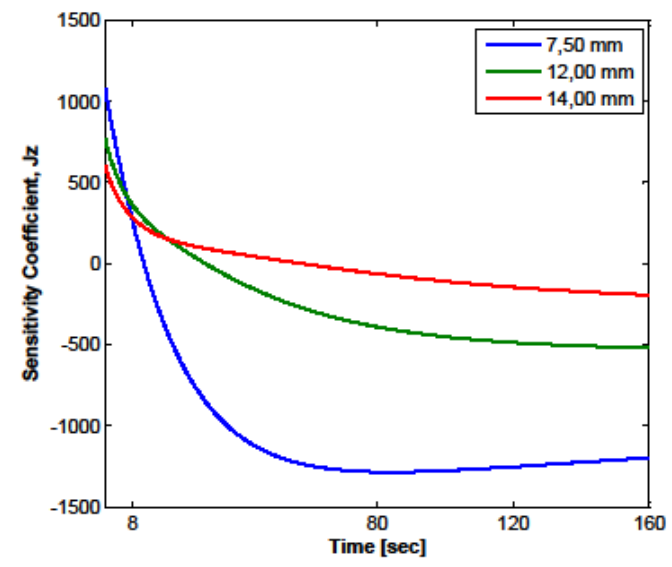

(a)

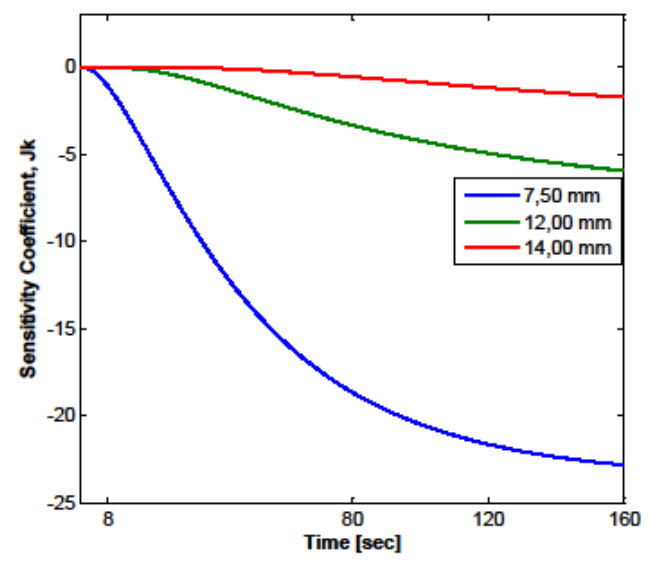

(b)

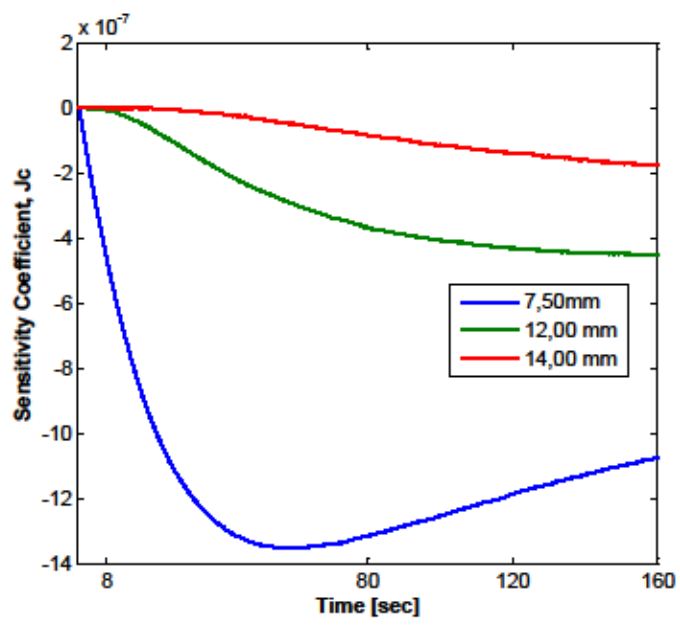

(c)

Fig. 2. Sensitivity coefficients for defects at different depth, considering properties (a) depth, (b) thermal conductivity and (c) heat capacity.

\section{REFERENCES}

[1] Lopez F. R., Nicolau V. P. and Bonin E., "Infrared thermography applied to ceramic materials: numerical analysis and experimental results" International Congress of Mechanical Engineering, Gramado (Brazil), 2009.

[2] Ozisik M. N. And Helcio R. B., Inverse Heat Transfer: Fundamentals and Applications, Taylor and Francis, (USA), 341 p., 2000. 